\title{
THE BIRDS OF THE LAST MOUNTAIN LAKE WILDLIFE AREA, SASKATCHEWAN
}

\author{
by Gary G. Anweiler, Silton, Saskatchewan
}

\begin{abstract}
The following briefly annotated list was prepared by Gary Anweiler and is based primarily on records collected by him between April 11 and October 27, 1969. During this period he was on contract with the Canadian Wildlife Service to collect natural history information for use at an interpretation centre scheduled to be built southeast of Simpson, Saskatchewan, within the next few years. The area investigated was approximately 3 miles wide and 12 miles long around the north end of Last Mountain Lake. (See Hatfield, J. P. 1969. The Last Mountain Lake Wildlife Area. Saskatchewan. Blue Jay $27: 129-131$.) It includes parts of Townships 27 and 28, Ranges 23 and 24 , west of the 2 nd Meridian. In the list, extreme dates of occurrence are given and, in some cases, peak migration dates. A date in parentheses is for a straggler. Locations given are usually those where the species was most commonly seen. Species marked with an asterisk are known to have bred in the area.

Information from other observers, mostly unpublished, is included and acknowledged (in parentheses). The publication referred to as "Todd" is W. E. C. Todd's Notes on the birds of southern Saskatchewan. Annals of the Carnegie Museum 30:383-421. 1947. The late Mr. Todd and G. M. Sutton worked in the area with A. C. Lloyd of Davidson, Saskatchewan, in May and June, 1932. Species marked "Not recorded by Todd" are ones that Anweiler found breeding or as summer visitors in the area.-J. B. Gollop, C.W.S., Saskatoon.
\end{abstract}

COMMON LOON. Migrant, rare summer visitor. Open water of the lake. April 28-June 25.

*RED-NECKED GREBE. Uncommon summer resident. Marshy bays of Lake. Taylor's Bay, bay inside Perry's Point, Fingers. May 26 (Todd) - June 22.

*HORNED GREBE. Migrant, rare summer resident. Marshy bays of lake, sloughs. May 1 - September 22.

EARED GREBE. Uncommon summer resident. Bays of lake, large sloughs. May 29 -September 18 (Sugré).

*W ESTERN GREBE. Common summer resident. Lake; especially in marshy bays. Taylor's Bay has a large breeding colony. May 4 - September 27.

*PIED-BILLED GREBE. Common summer resident. Marshy bays of lake, small deep marshes. Second Finger, Taylor's Bay. May 1 - September 28. Not recorded by Todd.
*WHITE PELICAN. Common summer visitor. Once bred (to 1954) but no longer does. Open water of lake, rocky islands. Island off Perry's Point. Dam at Ducks Unlimited project during last half of April. April 9 (Huggins) - October 26. Migration: April 14 - May 26; August 27 - September 30 .

*DOUBLE - CRESTED CORMORANT. Common summer resident. Breed on island off Perry's Point. $12,000 \pm$ noted leaving Finger on October 5, 1960 (Gollop). No concentration noted in 1969. April 23 - October 9.

GREAT BLUE HERON. Uncommon summer visitor. Reedy shore of the lake, Fingers shoreline near Watertown. April 11 - October 8 (one nest, Todd).

B L A C K - CROWNED NIGHT HERON. Uncommon summer visitor. Deeper marshes, Taylor's Bay, lakeshore, etc. Especially at slough north of gravel pits. May 23 - September 29.

*A M E R I CAN BITTERN. Fairly common summer resident. Reedy marshes, sloughs. Second Finger-deep marshes southwest of Headquarters. April 29 - October 13.

WHISTLING SWAN. Common migrant, casual summer visitor. Spring: Flooded sloughs in farm land along west and northwest edge of management area, as well as on the lake. April 12 (Huggins) - May 11 (May 17). Large flight on May 3. Fall: Fingers and open water of lake. September 22 October 27.

*CANADA GOOSE (Large). Uncommon summer resident. Lake, breeds on islands; Taylor's Bay. April 11 . September 27.

CANADA GOOSE (Small). Common spring migrant. Uncommon fall migrant. Spring. Rest on larger sloughs to west and northwest of management area; feeds in surrounding grain fields. Also found on lake in 
island area east of Watertown. March 22 (Huggins) - May 11. 6000-8000 at peak. Fall: Found with other geese (see White-fronted Goose). August 18 (Huggins) - September 27.

WHITE-FRONTED GOOSE. Very common migrant, spring and fall. Spring: Occur with small Canada Geese. April 11 - May 12 (May 28). Fall: Found in the island area of the lake; going out in all directions to feed, dawn and dusk, in the grain fields. September 12 - October 18.

SNOW GOOSE. Uncommon migrant, commonest in the fall. Found with the other geese. April 17 - May 11; September 13 - October 13.

BLUE GOOSE. Very uncommon lake; going out in all directions to feed, migrant. Usually with Snow Geese. April 21-24; October 9-13.

ROSS' GOOSE. Uncommon fall migrant. With other geese. September 13 (Chopping) - 18.

* M A L L A R D. Abundant summer resident. All wet places. Concentration in Fingers, especially Second, from mid-August to end-September; 100,000 estimated during this time. March 22 (Huggins) - October 27 - freeze-up.

BLACK D UCK. Rare summer visitor. Marshy bays and Fingers. June 2 - September 26. Not recorded by Todd.

*GADWALL. Fairly common summer resident. Alkali Lake behind Watertown, marshes, sloughs, etc. April 14 - October 16.

* PINTAIL. Common summer resident. Weedy places, shallows of lake. 15,000 at peak. April 10 (Hatfield) October 17.

*GREEN-WINGED TEAL. Common migrant; uncommon summer resident. Marshes, sloughs. April 14 - October 16. Common from: April 14-21; October 1-15.

*BLUE-WINGED TEAL. Common summer resident. Marshy shallows of lake. April 21 - October 6.

*AMERICAN WIDGEON. Common summer resident, common during migration. In April, in ponds and stubble fields, later in marshes espec- ially on lake, alkali slough behind Watertown. April 11 - October 25.

*SHOVELER. Common summer resident. Sloughs and marshes; Fingers. April 13 - October 17.

*REDHEAD. Fairly common summer resident. Deeper marshes and bays of the lake. Wherever there is thick bulrush growth. Second Finger, etc. April 14 - October 16.

RING-NECKED DUCK. Uncommon migrant. Deep open marshes and bays. Second Finger. April 25 - May 4; late August - September 27 (Hatfield).

*CANVASBACK. Fairly common migrant; uncommon summer resident. Marshes and bays of lake during summer and fall; temporary sloughs in April. Second Finger. April 14 - October 16. Migration: April 14 - early May; September 1 - October 16.

* LESSER SCAUP. Very common in migration; fairly common summer resident. Deep marshes; bays and open water of the lake. During spring migration found on larger temporary sloughs, often in rafts of up to 1,000 birds. April 14 - October 25. Spring Migration: April 14 - mid-May.

COMMON GOLDENEYE. Uncommon migrant and rather rare summer visitor. Open water of the lake. April 15 - May 4 (June 10); September 19 freeze-up.

BUFFLEHEAD. Fairly common migrant; very uncommon summer visitor. Open marshes and bays of the lake; larger sloughs. April 16 - October 25.

*WHITE-WINGED SCOTER. Not common summer resident. Open water of lake, especially near the islands; off beach at Watertown and south along shore to Taylor's Bay. Breed on islands. Large rafts of young (up to 74) noted on August 7 and 8. May 18 September 27.

*RUDDY DUCK. Not common summer resident. Deep marshes, bays and fingers of lake. Second Finger. April 29 - October 16.

HOODED MERGANSER. Very uncommon migrant; rare summer visitor. Ducks Unlimited Project, marshes of lake. May 23 (Todd) October 10 (Hatfield). 
COMMON MERGANSER. Uncommon migrant, open water of lake. April 19 - May 23.

RED-BREASTED M ER G A NSER. Fairly common migrant (Todd). Lake. May 24 (Todd) - June 5 (Todd). Not recorded in 1969.

SHARP-SHINNED HAWK. Uncommon migrant. Large shelterbelts, e.g. at Headquarters. April 12 - May 19; August 16 (?) - October 13.

COOPER'S HAWK. Rare migrant. August 25.

RED - TAILED HAWK. Common migrant; rare summer visitor. Migrations: April 11 - April 25; August 25 October 16 (October 26).

*SWAINSON'S HAWK. Common summer resident. Prairie areas, etc. April 21 - September 24.

ROUGH-LEGGED HAWK. Uncommon migrant. April 12 - May 1; August 20 - September 13.

*FERRUGINOUS HAWK. Very rare summer resident. Wreford Pasture. June 21 (nest).

GOLDEN EAGLE. Rare migrant. October 5 (Gollop) - October 13.

BALD EAGLE. Very uncommon migrant. April 12-13; October 13.

*MARSH HAWK. Very common summer resident. April 11 - October 25.

OSPREY. Rare migrant. April 30.
PRAIRIE FALCON. Migrant (Todd). May 24 (Todd) - June 3 (Todd). Not recorded in 1969.

PEREGRINE FALCON. Uncommon migrant. Marshes, especially Fingers. April 27 - May 28 (Todd); September 22 - October 16.

PIGEON HAWK. Very uncommon migrant. In vicinity of water. April 26; September 4-19.

SPARROW HAWK. Common migrant. Roadisides, or power and telephone lines and poles. April 14 - May 10; July 30 - September 23.

GREATER PRAIRIE CHICKEN. Probably now extinct in this area.

*SHARP-TAILED GROUSE. Fairly common resident. In fall, at dawn and dusk, in the stubble fields; during the day, in the willow swales. Prairie areas always good. April 25 - June 13 on dancing grounds. Broods June 21 - July 30 .

*GRAY PARTRIDGE. Fairly common year-round resident. Old shelterbelts, especially near cultivated areas. Feed in grain; dawn and late afternoon. Not recorded by Todd.

WHOOPING CRANE. Rare, but regular migrant, rare summer visitor. Fingers, bays of lake. With Sandhills.

SANDHILL CRANE. Abundant migrant, rare summer visitor. During day in grain fields or prairies around

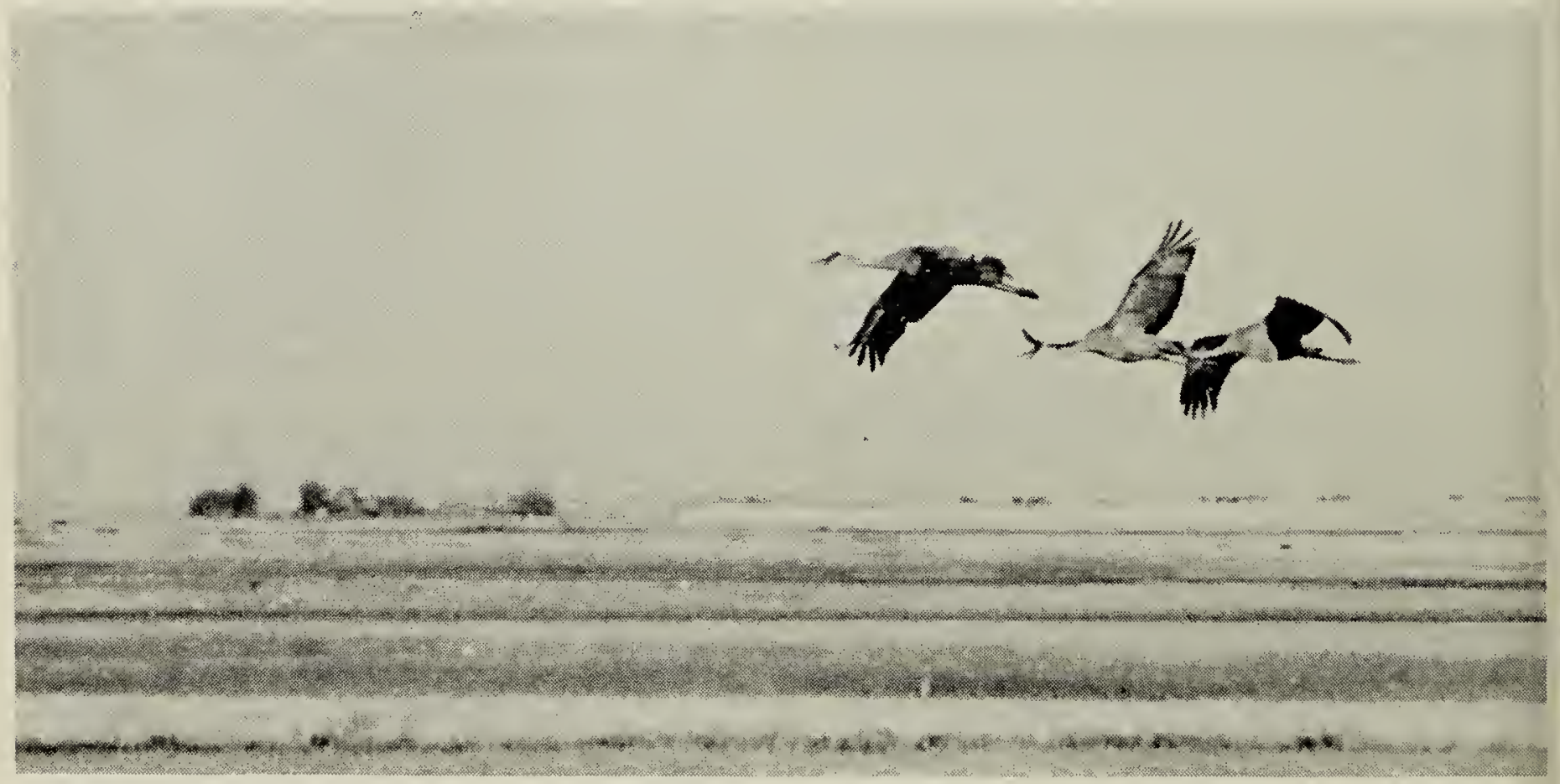

Sandhill Cranes in Last Mountain Lake Wildlife Area 


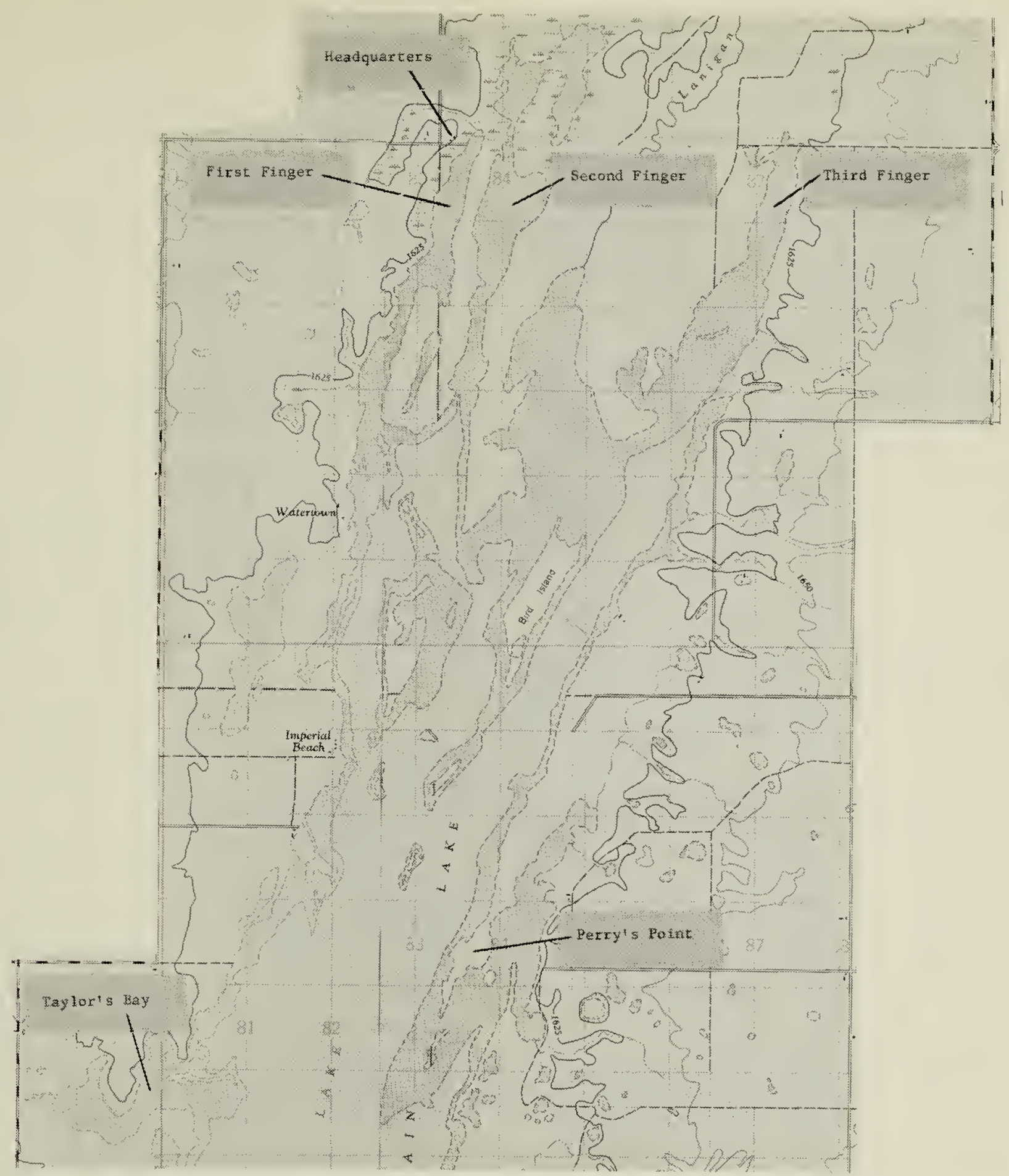

Part of Last Mountain Lake Wildlife Area. For a description of the area and for a map showing its boundaries see J. P. Hatfield's article, Blue Jay, September 1969, pp 129-131.

lake. Spring: Mostly west of management area. Fall: Mostly on game preserve. April 9 (a farmer) - May 1; July 31 - October 30 (Stephens).

VIRGINIA RAIL. Rare summer resident. Marshes. June 12 - August 7.

*SORA. Fairly common summer resident. Marshes (southwest of Headquarters). May 11 - September 9.

YELLOW RAIL. Summer resident (Todd). Grassy marshes, head of lake. May 28 (Todd) - June 25 (Todd). Not recorded in 1969 .
*AMERICAN COOT. Abundant summer resident. Found on all larger sloughs, marshes, and marshy areas of lake. Concentration noted on First Finger. Late August through September $(5,000 \pm$ here on September 20). April 22 - October 16.

SEMIPALMATED PLOVER. Uncommon migrant. Point $1 / 2$ mile south of Imperial Beach. Alkali lake behind Watertown. May 23 (Todd) - August 25.

*PIPING PLOVER. Rare summer 
resident. Alkali lake behind Watertown. June 2 (Todd) - July 17.

*KILLDEER. Uncommon summer resident. Usually found near water. April 11 - September 26.

AMERICAN GOLDEN PLOVER. Common spring migrant; rare fall migrant. Cultivated fields, especially those in fallow. May 17 - June 2; October 5 (Gollop).

BLACK-BELLIED PLOVER. Fairly common migrant, most common in spring. Beaches and mudflats. Point $1 / 2$ mile south of Imperial Beach, alkali lake behind Watertown. May 23 - June 5 (Bard \& Lahrman); July 1 - September 26.

RUDDY TURNSTONE. Uncommon migrant. Beaches, points, rocky islands. Watertown, point $1 / 2$ mile south cf Imperial Beach. May 22 (Todd) - June 22 (Todd) ; August $18-25$.

COMMON SNIPE. Uncommon summer resident, slightly commoner in migration. Apparently breeding around springs, southeast of the gravel pit, possibly at head of Third Finger. In migration, along edge of Second Finger. April 24 - October 16. Not recorded by Todd.

WHIMBREL. Rare migrant. May 27 (Todd) -30 (Todd). Not recorded in 1969.

*UPLAND PLOVER. Fairly common summer resident in prairie areas. Most common on flats between Second and Third Fingers. Also found in most pastures, especially those not overgrazed. Any prairie area. May 10 August 11 .

*SPOTTED SANDPIPER. Uncommon summer resident. Found on beaches, sandpoints and islands. Look for on Perry's Point, point $1 / 2$ mile south of Imperial Beach and in the vicinity of Watertown. May 23 August 25.

*WILLET. Common summer resident. Sloughs and marshy areas, lakeshore. Areas with a combination of prairie and water are most favourable. April 29 - September 12.
GREATER YELLOWLEGS. Fairly common in fall migration, rare in spring. Grassy and muddy shores. Along Fingers, Watertown, alkali lake behind Watertown. April 14 - May 18; July 3 - October 17.

LESSER YELLOWLEGS. Same as Greater Yellowlegs, but possibly slightly commoner. May 1-8; July 1 October 3.

KNOT. Uncommon migrant. Beaches, sandpoints. Point $1 / 2$ mile south of Imperial Beach. May 23 - June 7; August 2 and 4.

PECTORAL SANDPIPER. Uncommon migrant. Found along grassy or muddy shores. Alkali lake behind Watertown, lakeshore. May 14 (Todd)June 24 (22) (Todd); July 17 - October 16 .

WHITE-RUMPED SA N D PIPER. Uncommon spring migrant. Alkali lake behind Watertown. May 16 (Todd) June 23 (Todd).

BAIRD'S SANDPIPER. Same as Pectoral Sandpiper. Especially at head of Second Finger (?) June 4 (Todd) June 22 (Todd); August 25 -October 6.

LEAST SANDPIPER. Fairly common migrant. Grassy or muddy shores. Alkali lake behind Watertown, area around point $1 / 2$ mile south of Imperial Beach. May 14 (Todd) - June 11; July 1 - September 22.

SHORT-BILLED DOWITCHER and LONG-BILLED DOWITCHER. Common fall migrant, uncommon in spring. Muddy or grassy shallows. Especially around head of Second Finger. May 12 (Todd) - June 25 (Todd); July 1 October 16; September 19 - October 16. I collected two long-billed Dowitchers on September 23; Todd (1947) presumed his 11 specimens to be Shortbilled Dowitchers in 1932.

STILT SANDPIPER. Uncommon migrant. Muddy shores. Alkali lake behind Watertown. May 23 (Todd) June 13 (Todd); August 1 - September 9 .

SEMIPALMATED SA NDPIPER. Fairly common migrant. Muddy shores, other shores. Especially at alkali lake 
behind Watertown. May 22 (Todd) June 11; June 27 (Todd) - August 1.

BUFF - BREASTED SANDPIPER. Migrant (Todd). May 23 (Todd)-28 (Todd). Not recorded in 1969.

*M A R B LED GODWIT. Common summer resident. Found wherever there is prairie, especially near water, feeds along shores. April 28 - August 25.

SANDERLING. Uncommon migrant. Found on sandy beaches and points. Tip of Perry's Point, point $1 / 2$ mile south of Imperial Beach. May 23 - June 22 (Todd) August 18-25.

*AMERICAN AVOCET. Uncommon summer resident. Two colonies found at large alkali sloughs. Fifteen pair on Hatfield road, just east of the east boundary, about six pair at alkali slough behind Watertown. May 12 October 13.

*WILSON'S PHALAROPE. Fairly common summer resident. Grassy marsh edges, prairie shores. Especially along Second and Third Finger, Taylor's Bay. May 11 - August 10.

NORTHERN PHALAROPE. Fairly common migrant. Open water of large sloughs. Especially alkali lake behind Watertown. May 23 (Todd) - June 11; July 3; September 19 - October 3.

PARASITIC JAEGER. Accidental. October 28 (Bird, 1957).

HERRING GULL. Rare migrant and possible summer visitor. Along lake. May 16 (?) - 23 .

*CALIFORNIA GULL. Uncommon summer resident. Around lake. Few breed in Ring-billed colony off end of Perry's Point. April 20 - July 3.

*RING-BILLED GULL. Abundant summer resident. Three colonies. April 11 - October 25.

FRANKLIN'S GULL. Abundant summer visitor. Probably breeds at Kutawagon Lake. Found commonest near lake. Roost at alkali lake behind Watertown, Taylor's Bay. April 12 September 13.

BONAPARTE'S GULL. Uncommon migrant and summer visitor. Over open water of lake. May 23-July 23. Not recorded by Todd.

*FORSTER'S TERN. Uncommon summer resident. A few bred off mouth of Lanigan Creek and possibly in Second Finger also. June 1 - July 20. Not recorded by Todd.

*COMMON TERN. Abundant summer resident. Open water and along shores of the lake. Breeds on three small islands. May 3 - September 1.

BLACK TERN. Common summer resident, probably breeds. Marshy bays of lake, lakeshore, Taylor's Bay, Fingers, south side of point $1 / 2$ mile south of Imperial Beach. May 21 - September 9 .

* ROCK DOVE. Fairly common resident. Found around abandoned buildings, in which they breed. Not recorded by Todd.

MOURNING DOVE. Uncommon summer resident, probably breeds. Found in the shelterbelts. April 23October 5 .

*GREAT HORNED OWL. Uncommon resident year round. Aspen groves, shelterbelts.

SNOWY OWL. Uncommon migrant, probably winter visitor. To April 13.

*BURROWING OWL. Rare summer resident. Pastures. July 10 (nest).

*LONG - EARED OWL. Common summer resident. Found nesting in shelterbelts and aspen willow groves; also found in any treed place. April 11 - October 18.

*SHORT - EARED OWL. Common summer resident. Low prairie area, marsh edge, weedy fields. February 7 (Hatfield) - October 27.

COMMON NIGHTHAWK. Uncommon migrant. May 21 - June 6; August 20 - September 18.

RUBY-THROATED HUMMINGBIRD. Very uncommon migrant. Found in shelter-belts, gardens. May 27-28; August 18 -September 13. Not recorded by Todd. 
BELTED KINGFISHER. Very uncommon migrant. Lakeshore, along Lanigan Creek. April 28 - May 23.

*YELLOW - SHAFTED FLICKER. Common summer resident. All treed areas, especially shelterbelts. April 14 October 20.

YELLOW-BELLIED SAPSUCKER. Uncommon fall migrant. Treed places. September 29 - October 7.

HAIRY WOODPECKER. Same as Downy Woodpecker. October 26.

DOWNY W OODPECKER. Rare migrant, probably winter visitor. September 29.

*EASTERN KINGBIRD. Common summer resident. All treed and brushy places. May 15 - September 4.

*WESTERN KINGBIRD. Common summer resident. Shelterbelts, aspen groves. May 12 - August 28.

EASTERN PHOEBE. Rare spring migrant. April 16.

LEAST FLYCATCHER. Uncommon spring and rare fall migrant. Shelterbelts. May 15-29; August 27.

WESTERN WOOD PEWEE. Rare spring migrant. Shelterbelts. May 27 and 28.

*HORNED LARK. Common summer resident, abundant migrant. Prairie, cultivated fields, etc. Commonest where ground cover is light (e.g., over-grazed pasture, old fields). Area east of road to north boundary from Headquarters especially good. April 11 - October 18.

TREE SWALLOW. Fairly common migrant, rare summer resident. Shelterbelts, lakeshore. April 25-September 12 .

*BANK SW A LLOW. Uncommon summer resident. Gravel pit on north boundary. In swallow flocks in fall. May 20 - September 11.

*BARN SWALLOW. Common summer resident. Anywhere there are buildings. April 26 - October 13.

CLIFF SW A L LOW. Uncommon transient. May 18-30; August 7 - September 11.

PURPLE MARTIN. Rare transient. September 1. 29.

BLUE JAY. Accidental visitor. May

*BLACK-BILLED MAGPIE. Common resident. Shelterbelts.

*COMMON CROW. Common summer resident. Throughout, especially where trees. March 23 (Huggins) October 13.

BLACK-CAPPED CHICK ADEE. Winter visitor.

RED - B R E A STED NUTHATCH. Uncommon migrant. Shelterbelts. May 11 -16; August 25 - October 6.

BROWN CREEPER. Uncommon migrant (fall). September 30 - October 3.

*HOUSE WREN. Common resident. Shelterbelts, treed places. May 14 September 19.

LONG-BILLED MARSH WREN. Rare summer resident. June 15 - October 9 .

SHORT-BILLED MARSH WREN. Rare summer resident (Todd). Not recorded in 1969.

*CATBIRD. Rare summer resident. May 26 -June 28. Not recorded by Todd.

*BROWN THRASHER. Fairly common summer resident. Shelterbelts, aspen-willow groves, etc. May 5 - September 10.

AMERICAN ROBIN. Fairly common migrant. Shelterbelts, treed places, etc. April 11 - May 26; Augustt 25 October 16.

HERMIT THRUSH. Fairly common migrant (fall). Shelterbelts, treed places. October 1-14.

SW A I NSON'S THRUSH. Fairly common migrant. Shelterbelts, treed places. May 8 - June 2; September 10 October 9.

GRAY-CHEEKED THRUSH. Fairly common migrant. Shelterbelts, treed places. May 8 - June 2; September 10 October 9.

VEERY. Rare summer visitor. Shelterbelts. May 16 - July 9. Not recorded by Todd.

MOUNTAIN BLUEBIRD. Rare migrant. Roadsides. April 13 - May 3 (Hatfield). 
TOWNSEND'S SOLITAIRE. Accidental summer visitor. September 24.

GOLDEN - CROWNED KINGLET. Uncommon migrant. Shelterbelts. April 30 (Todd); September 30 - October 19.

RUBY-CROWNED KINGLET. Uncommon migrant. Shelterbelts. May 15 and 16; September 10 - October 6 .

*SPRAGUE'S PIPIT. Common summer resident. Dry prairie areas. April 22 - September 18.

BOHEMIAN WAXWING. Migrant, probably winter months. Shelterbelts. April 13 and 17.

CEDAR WAXWING. Fairly common migrant and summer visitor. Shelterbelts. May 28 - September 28.

NORTHERN SHRIKE. Winter visitor. Treed places, roadsides. March 24 (Hatfield); early October.

*LOGGERHEAD SHRIKE. Fairly common summer resident. Shelterbelts, roadsides, any treed place. April 27 September 2.

*COMMON STARLING. Common summer resident. Shelterbelts. April 11 - October. Not recorded by Todd.

SOLITARY VIREO. Rare migrant. Shelterbelts. May 16.

RED - E Y E D VIREO. Uncommon migrant. Shelterbelts. May 27 - June 8.

PHILADELPHIA VIREO. Hypothetical (identification not positive). Shelterbelts.

WARBLING VIREO. Rare summer resident. Shelterbelts. May 25 -June 17.

BLACK - AND - WHITE WARBLER. Rare migrant. Shelterbelts. May 11 and September 11.

TENNESSEE WARBLER. Uncommon migrant and casual summer visitor. May 20-29; July 1-9; August 10-12 (?).

ORANGE-CROWNED WARBLER. Uncommon migrant. Shelterbelts. May 6-19; September 10 - October 6 .

*YELLOW W A R B LER. Common summer resident. Shelterbelts, any treed or brushy place. May $15-$ September 17.
MYRTLE WARBLER. Common migrant. Shelterbelts, aspen-willow groves. April 25-May 23 (Todd); August 25 - October 16.

BLACKPOLL WARBLER. Uncommon migrant. Shelterbelts. May 15-28; August 17.

PALM WARBLER. Very uncommon migrant (fall). Shelterbelts. September 10-22.

OVENBIRD. Rare migrant. Shelterbelts. May 15 - August 26.

MOURNING WARBLER. One trapped. September 10.

COMMON YELLOWTHROAT. Uncommon summer resident. Brushy marsh borders, shelterbelts, cattails. May 20 (Todd) - September 23.

WILSON'S WARBLER. Very uncommon migrant (fall). Shelterbelts. August 25 - September 17.

CANADA WARBLER. Rare migrant (fall). Shelterbelts. August 25 - September 10 .

A M ERICAN REDSTART. Two birds. May 27 and 28; September 21.

*HOUSE SPARROW: Fairly common resident. Occupies shelterbelts, old buildings (Headquarters). Not recorded by Todd.

B O B OLINK. Uncommon summer resident. Hay-meadows, slough borders. Along Lanigan Creek, especially lower end. May 19 - July 16.

*WESTERN MEADOWLARK. Common summer resident. Any area, roadsides are good. April 9 (Hatfield) October 25.

*YELLOW-HEADED BLACKBIRD. Common summer resident. Deep marshes. April 24 - October 9.

*RED-WINGED BLACKBIRD. Common summer resident. Any slough. April 11 - October 26.

*BALTIMORE ORIOLE. Uncommon summer resident. Shelterbelts. May 19 - August 26.

RUSTY BLACKBIRD. Rare migrant. September 18 (Sugré).

*BREWER'S BLACKBIRD. Common summer resident. Shelterbelts. April 20 - October 9. 
COMMON GRACKLE. Uncommon migrant, rare summer resident. Shelterbelts. April 14 - September 29.

*BROWN - HEADED COW B IRD. Common summer resident. Throughout. April 30 - August 25.

ROSE-B REA STE D GROSBEAK. Uncommon migrant. Shelterbelts. May 16 and 19.

PINE GROSBEAK. Winter visitor. Shelterbelts. October 26.

C O M ON REDPOLL. Winter visitor. Shelterbelts, weedy places. October 6 - April 11.

PINE SISKIN. Erratic (?) summer visitor. Shelterbelts, especially where spruce are present, such as at Headquarters. July 1 - October 8. Not recorded by Todd.

AMERICAN GOLDFINCH. Uncommon summer resident. Shelterbelts. May 19 - September 24.

WHITE-WINGED CROSSBILL. Uncommon and probably erratic visitor. Shelterbelts, especially where there are spruce. June 27 - October 14. Not recorded by Todd.

LARK BUNTING. June 2-15.

*SAVANNAH SPARROW. Common summer resident. Long grass, especially in moist places such as lakeshores and slough margins. April 23 - October 6.

*B A IRD'S SPARROW. Common summer resident. Dry prairie. May 3 August 1. June 23 (nest, Todd).

LECONTE'S SPARROW. Fairly common summer resident. May 5Aug. 8.

*SHARP-TAILED SPARROW. Rare (?) summer resident. Around marshes. June 13, nest (Todd) - July 21.

*VESPER SPARROW. Fairly common summer resident. April 23 - October 1 .

SLATE-COLORED JUNCO. Very common migrant. Wherever there are trees. April 11-28; September 12October 19.
TREE SPARROW. Abundant migrant. Brushy, weedy, and treed places. April 11 - 25; September 22 - October 27.

CHIPPING SPARROW. Fairly common migrant, rare summer visitor. Shelterbelts, treed places. May 6-28; July 15-21; September 11- 12 .

*CLAY - COLORED S P A R R O W. Common summer resident. Bushy or treed areas. Snowberry patches on prairie. Headquarters. May 4 - September 19 .

HARRIS'S SPARROW. Fairly common migrant. Treed places. May 7-15; September 10 - October 15.

WHITE - CROWNED SPARROW. Same as above. April 25 - May 16; September 12 - October 13.

WHITE-THROATED SPARROW. Common migrant. Treed places. April 30 - May18; September 10 - October 16.

FOX SPARROW. Uncommon migrant. Treed areas. May 1; September 30 - October 10.

LINCOLN'S SPARROW. Uncommon migrant. Treed places. May 4-16; September 11 -October 6 .

SWAMP SPARROW. Same as above. May 19; September 20 -October 6 .

SONG SPARROW. Uncommon migrant, rare summer resident. Shelterbelts. April 11 - July 9 .

McCOWN'S LONGSPUR. Local summer resident (Todd). Not recorded in 1969.

LAPLAND LONGSPUR. Abundant migrant. Any cultivated field. April 11 - May 24; September 9 - October 24.

*CHESTNUT - COLLARED LONGSPUR. Common summer resident. Dry prairie, grazed pasture. Along trail between Second and Third Fingers, from north boundary. April 11-July 19.

SNOW BUNTING. Migrant and winter visitor. Cultivated fields; with longspurs in spring. April 11-28; October 5 (Gollop) - 24 . 


\section{REFERENCES}

Bard, Fred G. and Fred W. Lahrman. 1969. Some observations of the Knot on Old Wives and Last Mountain Lakes. Blue Jay, $27: 210$.

Bird, C. D. 1957. The Parasitic Jaegar in Saskatchewan. Can. Field-Naturalist, 71-37.

Chopping, George. 1961. Ross' Geese at Last Mountain Lake. Blue Jay, 19:165.

Hatfield, John P. 1969. Unpublished field notes kept by Manager of Last Mountain Wildlife Area.
Huggins. ca. 1927-1931. Unpublished records kept by him while Game Guardian in the area.

Gollop, J. B. 1960. Unpublished field notes in C.W.S. files.

Stephens, W. J. D. 1967. Bionomics of the Sandhill Crane. C.W.S. Special Report Series - No. 2. Queen's Printer, Ottawa.

Sugré. ca. 1917. Letter to Munro in C.W.S. files.

Todd, W. E. C. 1947. Notes on the birds of southern Saskatchewan. Annals of the Carnegie Museum $30: 383-421$.

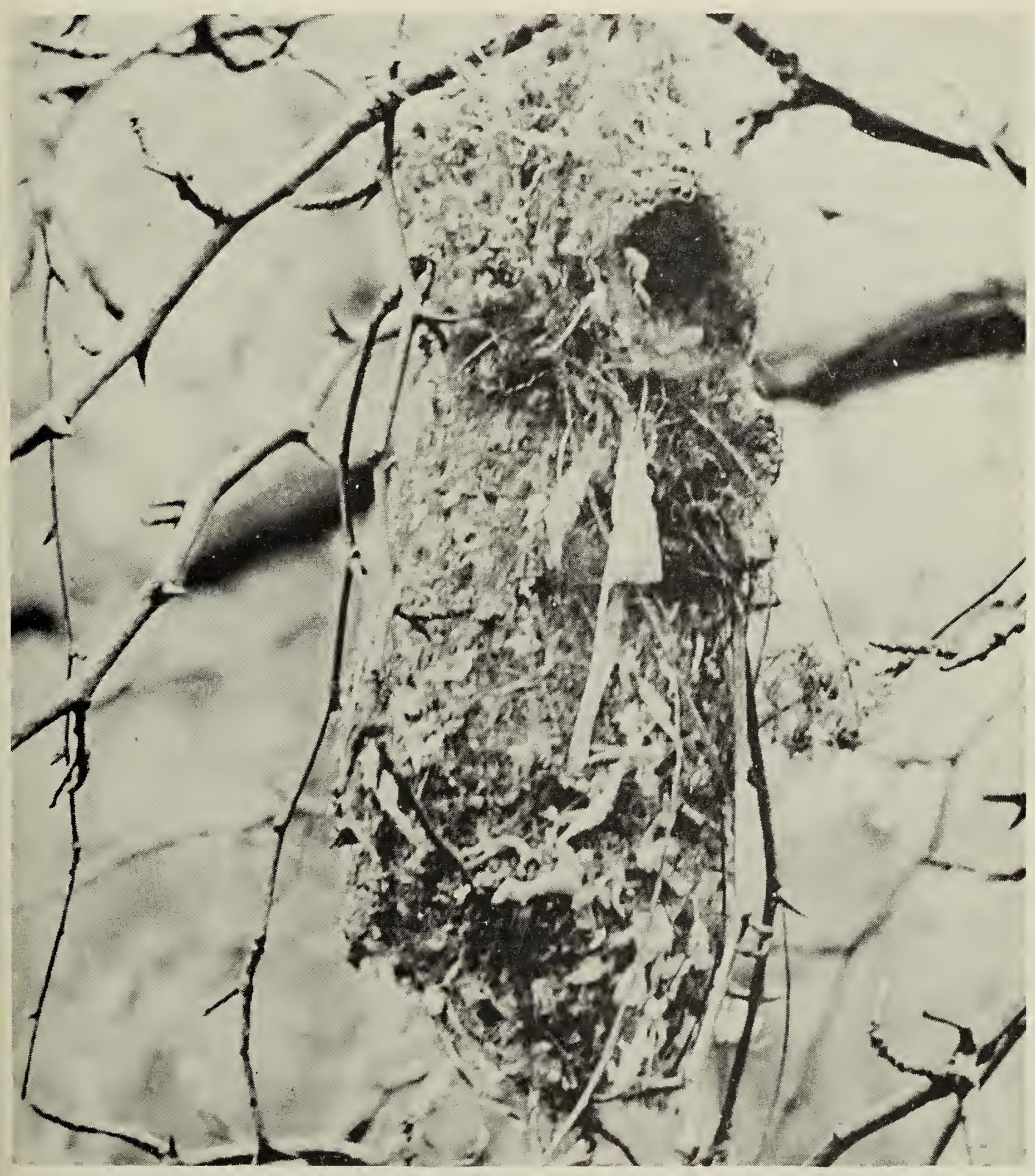

Photo by Al Grass, Manning Park

Common Bushtit nest, photographed near Ladner, B.C., May 1969. Nesting materials included mosses, twigs, spider webbing, grass and tissue p\&per. The nest was built in a hawthorn bush. 